\title{
On global solution, energy decay and blow-up for 2-D Kirchhoff equation with exponential terms
}

\section{Gongwei Liu*}

\section{"Correspondence:}

gongweiliu@126.com

Department of Mathematics, Henan

University of Technology,

Zhengzhou, 450052, China

School of Mathematics and

Statistics, Wuhan University, Wuhan,

430072, China

\section{Springer}

\begin{abstract}
This paper is concerned with the study of damped wave equation of Kirchhoff type $u_{t t}-M\left(\|\nabla u(t)\|_{2}^{2}\right) \Delta u+u_{t}=g(u)$ in $\Omega \times(0, \infty)$, with initial and Dirichlet boundary condition, where $\Omega$ is a bounded domain of $\mathbb{R}^{2}$ having a smooth boundary $\partial \Omega$. Under the assumption that $g$ is a function with exponential growth at infinity, we prove global existence and the decay property as well as blow-up of solutions in finite time under suitable conditions.
\end{abstract}

MSC: 35L70; 35B40; 35B44

Keywords: exponential source; Kirchhoff equation; global existence; energy decay; blow-up

\section{Introduction}

Let $\Omega$ be a bounded domain with smooth boundary $\partial \Omega$, we are concerned with the initialboundary value problem

$$
\left\{\begin{array}{l}
u_{t t}-M\left(\|\nabla u(t)\|_{2}^{2}\right) \Delta u+u_{t}=g(u) \quad \text { in } \Omega \times(0, \infty), \\
u(0, x)=u_{0}(x), \quad u_{t}(0, x)=u_{1}(x), \quad x \in \Omega, \\
u(t, x)=0 \quad \text { on } \partial \Omega \times(0, \infty),
\end{array}\right.
$$

where $g$ is a source term with exponential growth at the infinity to be specified later, $M(s)$ is a positive $C^{1}$ class function in $s \geq 0$. It is said that (1.1) is non-degenerate if there exists a constant $m_{0}>0$ such that $M(s) \geq m_{0}$ for all $s \geq 0$. If there exists a point $s_{0} \geq 0$ such that $M\left(s_{0}\right)=0$, then it is said that (1.1) is degenerate. In the case $M(s) \equiv m_{0}>0,(1.1)$ is usually a semilinear wave equation. In this paper, we only consider non-degenerate case.

It is known that Kirchhoff [1] first investigated the following nonlinear vibration of an elastic string for $\delta=f=0$ :

$$
\rho h \frac{\partial^{2} u}{\partial t^{2}}+\delta \frac{\partial u}{\partial t}=\left\{p_{0}+\frac{E h}{2 L} \int_{0}^{L}\left(\frac{\partial u}{\partial x}\right)^{2} d x\right\} \frac{\partial^{2} u}{\partial x^{2}}+f ; \quad 0 \leq x \leq L, t \geq 0
$$

where $u=u(x, t)$ is the lateral displacement at the space coordinate $x$ and the time $t, \rho$ the mass density, $h$ the cross-section area, $L$ the length, $E$ the Young modulus, $p_{0}$ the initial axial tension, $\delta$ the resistance modulus, and $f$ the external force.

(C) 2014 Liu; licensee Springer. This is an Open Access article distributed under the terms of the Creative Commons Attribution License (http://creativecommons.org/licenses/by/4.0), which permits unrestricted use, distribution, and reproduction in any medium, provided the original work is properly credited. 
Recently, Alves and Cavalcanti [2] studied the following problem with nonlinear damping term:

$$
\left\{\begin{array}{l}
u_{t t}-\Delta u+h\left(u_{t}\right)=g(u) \quad \text { in } \Omega \times(0, \infty), \\
u(0, x)=u_{0}(x), \quad u_{t}(0, x)=u_{1}(x), \quad x \in \Omega, \\
u(t, x)=0 \quad \text { on } \partial \Omega \times(0, \infty),
\end{array}\right.
$$

where $g$ is a source term with exponential growth at infinity to be specified later, $h(\cdot)$ is a monotone continuous function with polynomial growth at infinity and with no restriction on the growth rate near the origin. There are few works in the literature dealing with the exponential source even for wave equation, the work [2] is a recent one in this direction. In [3] Ma and Soriano studied an evolution equation with exponential term of the following form:

$$
u_{t t}-\operatorname{div}\left(|\nabla u|^{n-2} \nabla u\right)-\Delta u_{t}+g(u)=f(t, x) \quad \text { in } \Omega \times(0, \infty),
$$

with initial and Dirichlet boundary condition, where $\Omega \subset \mathbb{R}^{n}$ is a bounded domain with smooth boundary $\partial \Omega, n \geq 2, g(u)$ grows like $e^{|u|^{\frac{n}{n-1}}}$ and satisfies the sign condition $g(u) u \geq 0$. More recently, Han and Wang [4] studied the following problem:

$$
u_{t t}-\Delta u-\omega \Delta u_{t}+\mu u_{t}=g(u) \text { in } \Omega \times(0, \infty)
$$

with initial and Dirichlet boundary condition, where $\Omega \subset \mathbb{R}^{2}$ is a bounded domain with smooth boundary $\partial \Omega, g(u)$ is just the term considered in [2]. In fact, when $g(u)=|u|^{p-2} u$, the problem (1.3) was studied by Gazzola and Squassina in [5].

To the author's knowledge, there are few works in the literature dealing with the exponential source for Kirchhoff equations. When the source term $g(u)$ is a nonlinear function like $\pm|u|^{\alpha} u$ for $\alpha \geq 0$, the problem (1.1) has been discussed by many authors; see [6-10] and the references cited therein.

Motivated by there papers, in this study, we concentrate on studying the problem (1.1) with $M(s) \geq m_{0}>0$ for constant $m_{0}$. In what follows, we would like to introduce some wellknown theory of elliptic problems. More precisely, defining the functional $\tilde{J}(\cdot): H_{0}^{1}(\Omega) \rightarrow$ $\mathbb{R}$ by

$$
\tilde{J}(u)=\frac{m_{0}}{2} \int_{\Omega}|\nabla u|^{2} d x-\int_{\Omega} G(u) d x,
$$

where $G(u)=\int_{0}^{u} g(s) d s$. The critical points of the functional $\tilde{J}$ are the weak solutions of the elliptic problem

$$
\left\{\begin{array}{l}
-m_{0} \Delta u=g(u) \quad \text { in } \Omega \\
u(x)=0 \quad \text { on } \partial \Omega .
\end{array}\right.
$$

Defining the functional $\tilde{I}(\cdot): H_{0}^{1}(\Omega) \rightarrow \mathbb{R}$ by

$$
\tilde{I}(u)=m_{0}\|\nabla u\|_{2}^{2}-\int_{\Omega} g(u) u d x .
$$


Related to the functional $\tilde{J}$, we have the well-known Nehari manifold:

$$
\mathcal{N}=\left\{u \mid u \in H_{0}^{1}(\Omega) \backslash\{0\}: \tilde{I}(u)=0\right\} .
$$

If $g$ satisfies some suitable properties, it is possible to prove the functional $\tilde{J}$ satisfies the hypotheses of the mountain pass theorem due to Ambrosetti and Rabinowitz [11], and the level

$$
d=\inf _{u \in H_{0}^{1}(\Omega) \backslash\{0\}} \max _{\lambda \geq 0} \tilde{J}(\lambda u)>0
$$

called mountain pass level is a critical level for $\tilde{J}$. By Theorem 4.2 in [12], the mountain pass level $d$ can be characterized as

$$
d=\inf _{u \in \mathcal{N}} \tilde{J}(u)
$$

In order to study the problem (1.1), we define some additional functionals. Define

$$
\begin{aligned}
& J(u)=\frac{1}{2} \bar{M}\left(\|\nabla u\|_{2}^{2}\right)-\int_{\Omega} G(u) d x, \\
& I(u)=M\left(\|\nabla u\|_{2}^{2}\right)\|\nabla u\|_{2}^{2}-\int_{\Omega} g(u) u d x,
\end{aligned}
$$

where $\bar{M}(r)=\int_{0}^{r} M(s) d s$. Then we can define

$$
W=\left\{u \mid u \in H_{0}^{1}(\Omega): \tilde{I}(u)>0\right\} \cup\{0\} .
$$

Now, as usual setting

$$
E(t)=\frac{1}{2}\left\|u_{t}(t)\right\|_{2}^{2}+J(u(t)) .
$$

The remainder of this paper is organized as follows. Section 2 is concerned with some notation, statement of assumptions and the main results. Sections 3 and 4 are devoted to the proofs of the main results.

\section{Assumptions and main results}

To state our results, we need the following assumptions.

(A1) Assume that $g: \mathbb{R} \rightarrow \mathbb{R}$ is a $C^{1}$ function satisfying:

- For each $\beta>0$, there exists a positive constant $C_{\beta}$ such that

$$
\left|g^{\prime}(\zeta)\right|,|g(\zeta)| \leq C_{\beta} e^{\beta \zeta^{2}}, \quad \text { for all } \zeta \in \mathbb{R} .
$$

- Near the origin we have

$$
\lim _{\zeta \rightarrow 0} \frac{g(\zeta)}{\zeta}=0
$$

- The function $g(\zeta) / \zeta$ is increasing in $(0, \infty)$. 
(A2) There exists a positive constant $\theta>2$ such that

$$
0<\theta G(\zeta)<g(\zeta) \zeta, \quad \text { for all } \zeta \in \mathbb{R} \backslash\{0\}
$$

A typical example of functions satisfying (A1) is

$$
g(\zeta)=|\zeta|^{p-2} \zeta e^{C|\zeta|^{\alpha}}, \quad \text { for all } \zeta \in \mathbb{R}
$$

where $p>2, C>0, \alpha \in(1,2)$ arbitrarily chosen. From (2.2), for each $\varepsilon>0$ fixed, there exists $\delta>0$ such that

$$
|g(\zeta)| \leq \varepsilon|\zeta|, \quad \text { for all } \zeta \in[-\delta, \delta]
$$

Moreover, from (2.1), for each $\beta>0$ and $p \geq 1$ fixed, there exists $C_{\beta}>0$ such that

$$
|g(\zeta)| \leq \delta^{-p+1} C_{\beta}|\zeta|^{p-1} e^{\beta \zeta^{2}}, \quad \text { for all } \zeta \in(-\infty,-\delta] \cup[\delta,+\infty)
$$

Hence, for each $\beta, \varepsilon>0$ and $p \geq 1$ fixed, there exists $\delta$ and $C_{\beta, \varepsilon, p}>0$ satisfying

$$
\begin{aligned}
& |g(\zeta)| \leq \varepsilon|\zeta|+C_{\beta, \varepsilon, p}|\zeta|^{p-1} e^{\beta \zeta^{2}}, \quad \text { for all } \zeta \in \mathbb{R}, \\
& |G(\zeta)| \leq \frac{\varepsilon}{2}|\zeta|^{2}+C_{\beta, \varepsilon, p}|\zeta|^{p} e^{\beta \zeta^{2}}, \quad \text { for all } \zeta \in \mathbb{R}
\end{aligned}
$$

where $G(\zeta)=\int_{0}^{\zeta} g(s) d s$

Remark 2.1 The assumptions (A1) and (A2) have been used in [2]. The condition (2.3) is the well-known Ambrosetti-Rabinowitz condition, widely used in elliptic problem. Also as remarked in $[2,12]$, the mountain pass level $d$ can be characterized by (1.6) provided (2.1), (2.2), and (2.3) hold.

Throughout this paper we will make use of the Moser-Trudinger inequality found in $[13,14]$.

Lemma 2.1 Let $\Omega$ be a bounded domain in $\mathbb{R}^{n}, n \geq 2$. For all $u \in W_{0}^{1, n}(\Omega)$,

$$
e^{\alpha|u|^{\frac{n}{n-1}}} \in L^{1}(\Omega), \quad \text { for all } \alpha>0,
$$

and there exist positive constants $C_{n}$ and $\alpha_{n}$ such that

$$
\sup _{\|u\|_{W_{0}^{1, n}(\Omega)} \leq 1} \int_{\Omega} e^{\alpha|u|^{\frac{n}{n-1}}} d x \leq C_{n}, \quad \text { for all } \alpha \leq \alpha_{n},
$$

where $\alpha_{n}=n \omega_{n-1}^{1 /(n-1)}$ and $\omega_{n-1}$ is the $(n-1)$-dimensional surface of unit sphere, specially, $\alpha_{2}=4 \pi$.

Now, we state our main results. First, we consider the problem (1.1) with $M(s)=1+s^{\gamma / 2}$ for $\gamma>1$. We have the following global existence and decay result. 
Theorem 2.1 Assume that (A1) and (A2) hold, $M(s)=1+s^{\gamma / 2}$ for $\gamma>1$. Then there exists an open set $S$ in $\left(W \cap H^{2}(\Omega)\right) \times H_{0}^{1}(\Omega)$, which contains $(0,0)$, if $\left(u_{0}, u_{1}\right) \in S$ and the initial energy $E(0)<d$, then $u(t) \in W$ on $[0, \infty)$. Furthermore, suppose that there exists a constant $\eta_{0} \in(0,1)$ such that

$$
\tilde{I}(u(t)) \geq \eta_{0}\|\nabla u(t)\|_{2}^{2}, \quad \text { for } t \geq 0,
$$

then the problem (1.1) has a unique solution $u=u(t)$ satisfying

$$
u \in L^{\infty}\left(\mathbb{R}^{+} ; H_{0}^{1}(\Omega) \cap H^{2}(\Omega)\right) \cap W^{1, \infty}\left(\mathbb{R}^{+} ; H_{0}^{1}(\Omega)\right) \cap W^{2, \infty}\left(\mathbb{R}^{+} ; L^{2}(\Omega)\right) .
$$

Furthermore, we have the following energy decay estimate:

$$
E(t) \leq E(0) e^{-\kappa[t-1]^{+}} \text {on }[0, \infty)
$$

where $\kappa$ is a positive constant.

Secondly, we consider the initial-boundary value problem (1.1) under the following general assumption.

(A3) Assume that the sign condition $g(s) s \leq 0$ holds for all $s \in \mathbb{R}$. $M(s)$ is a positive $C^{1}$ function on $[0, \infty)$, and

$$
M(s) \geq 1, \quad\left|M^{\prime}(s)\right| \leq s^{\alpha}, \quad \text { for } \alpha \geq 0 .
$$

Then we can state the global existence and energy decay to the related problem (1.1).

Theorem 2.2 Let (A1) and (A3) hold, then there exists an open set $\operatorname{Sin}\left(H_{0}^{1}(\Omega) \cap H^{2}(\Omega)\right) \times$ $H_{0}^{1}(\Omega)$, which contains $(0,0)$ such that if $\left(u_{0}, u_{1}\right) \in S$, then the conclusions of Theorem 2.1 hold.

Our final result is concerned with the blow-up phenomenon. First of all we give the following assumption.

(A4) There exists a positive constant $\delta$ such that

$$
s g(s) \geq(2+4 \delta) G(s), \quad \text { for all } s \in \mathbb{R}
$$

and

$$
(2 \delta+1) \bar{M}(s)-M(s) s \geq 0, \quad \text { for all } s \geq 0 .
$$

Theorem 2.3 Under the assumptions (A1) and (A4), and that either one of the following conditions is satisfied:

(i) $E(0)<0$,

(ii) $E(0)=0$ and $\int_{\Omega} u_{0} u_{1} d x>0$,

(iii) $0<E(0)<\frac{\left(\int_{\Omega} u_{0} u_{1} d x\right)^{2}}{2\left(T_{1}+1\right)\left\|u_{0}\right\|_{2}^{2}}$ and (4.10) holds, where $T_{1}$ to be chosen later.

Then the solution $u$ blows up at finite $T^{*}$. And $T^{*}$ can be estimated by (4.19)-(4.23), respectively, according to the sign of $E(0)$. 


\section{Global existence and energy decay}

In this section, we will give the solvability in the class of $H^{2}(\Omega) \cap H_{0}^{1}(\Omega)$ and the energy decay of the problem (1.1). From now on we denote $c$ or $c_{i}$ various positive constants.

\subsection{Proof of Theorem 2.1}

In this section we take $M(s)=1+s^{\frac{\gamma}{2}}$ for $\gamma>1$, and $u_{0} \in W \cap H^{2}(\Omega)$ and $u_{1} \in H_{0}^{1}(\Omega)$. We employ the Galerkin method to construct a global solution. Let $\left\{\lambda_{i}\right\}_{i=1}^{\infty}$ be a sequence of eigenvalues for $-\triangle w=\lambda w$ in $\Omega$ and $w=0$ on $\partial \Omega$. Let $w_{i} \in H_{0}^{1}(\Omega) \cap H^{2}(\Omega)$ be the corresponding eigenfunction to $\lambda_{i}$ and take $\left\{w_{i}\right\}_{i=1}^{\infty}$ as a completely orthonormal system in $L^{2}(\Omega)$. We construct approximate solutions $u_{m}$ in the form $u_{m}(t)=\sum_{i}^{m} g_{i m}(t) w_{i}$, where $g_{i m}$ are determined by the following ordinary differential equations:

$$
\left\{\begin{array}{l}
\left(u_{m}^{\prime \prime}(t), w_{i}\right)+M\left(\left\|\nabla u_{m}(t)\right\|_{2}^{2}\right)\left(\nabla u(t), \nabla w_{i}\right)+\left(u_{m}^{\prime}(t), w_{i}\right) \\
\quad=\left(g\left(u_{m}(t)\right), w_{i}\right), \quad i=1, \ldots, m, \\
u_{m}(0)=u_{0 m}=\sum_{i=1}^{m}\left(u_{0}, w_{i}\right) w_{i} \rightarrow u_{0} \quad \text { as } m \rightarrow \infty \text { in } H_{0}^{1}(\Omega) \cap H^{2}(\Omega), \\
u_{m}^{\prime}(0)=u_{1 m}=\sum_{i=1}^{m}\left(u_{1}, w_{i}\right) w_{i} \rightarrow u_{1} \quad \text { as } m \rightarrow \infty \text { in } H_{0}^{1}(\Omega) .
\end{array}\right.
$$

System (3.1) can easily be solved by Picard's iteration method, hence it admits a local solution on some interval $\left[0, T_{m}\right)$ with $0<T_{m} \leq \infty$. Note that $u_{m}(t)$ is of $C^{2}$ class. We shall see that $u_{m}(t)$ can be extended to $[0, \infty)$, which needs some prior estimates for $u_{m}(t)$. But this procedure allows us to employ the energy method for an assumed smooth solution $u(t)$ to the problem (1.1) (the results should be in fact applied to approximated solutions).

Now, it is easy to see the following energy identity:

$$
E(t)+\int_{0}^{t}\left\|u_{t}(s)\right\|_{2}^{2} d s=E(0)
$$

as long as the approximated solutions exist. First we discuss the $H^{1}$ a priori estimate.

Lemma 3.1 ( $H^{1}$ a priori bounds) Let $u(t)$ be a solution with the initial data $u_{0} \in$ $W \cap H^{2}(\Omega), u_{1} \in H_{0}^{1}(\Omega)$, and the initial energy $E(0)<d$. And the assumptions (A1) and (A2) hold. Then $u(t) \in W$ on $[0, \infty)$. Furthermore, there exists a constant $C=C\left(\left\|u_{1}\right\|_{2}\right.$, $\left.\left\|\nabla u_{0}\right\|_{2}\right)>0$ such that

$$
\left\|u_{t}(t)\right\|_{2}+\|\nabla u(t)\|_{2}^{2} \leq C, \quad \int_{0}^{t}\left\|u_{t}(s)\right\|_{2}^{2} d s \leq C,
$$

for all $t \geq 0$.

Proof Since $\tilde{J}(u(t)) \leq J(u(t))$, it follows from the energy identity (3.2) and the initial energy $E(0)<d$ that

$$
\frac{1}{2}\left\|u_{t}\right\|_{2}^{2}+\tilde{J}(u(t))+\int_{0}^{t}\left\|u_{t}(s)\right\|_{2}^{2} d s \leq E(0)<d, \quad \text { for all } t \in\left[0, T_{m}\right),
$$

which implies $\tilde{J}(u(t))<d$ for all $t \in\left[0, T_{m}\right)$. As in [2] arguing by contradiction, we can obtain $u(t) \in W$ on $\left[0, T_{m}\right)$. From this fact and (3.3), we can conclude that

$$
\int_{\Omega}\left(\frac{1}{2} g(u) u-G(u)\right) d x<d
$$


which together with the Ambrosetti-Rabinowitz condition (2.3) implies

$$
\int_{\Omega} G(u(t)) d x<\frac{2 d}{\theta-2} .
$$

Combining (3.3) and (3.4) we obtain

$$
\left\|u_{t}(t)\right\|_{2}^{2}+\|\nabla u(t)\|_{2}^{2}+2 \int_{0}^{t}\left\|u_{t}(s)\right\|_{2}^{2} d s \leq E(0)+\int_{\Omega} G(u(t)) \leq \frac{2 \theta d}{\theta-2},
$$

for all $t \in\left[0, T_{m}\right)$. At the same time, these estimates imply that the (approximated) solution $u(t)$ can be extended to the whole interval $[0, \infty)$. This concludes the proof of Lemma 3.1.

Moreover, since $u_{0} \in W$, we have $u(t) \in W$ for all $t \geq 0$ from Lemma 3.1. If $\tilde{I}(u)>0$, using (2.3), we have

$$
\begin{aligned}
E(t) & \geq \frac{1}{2}\left\|u_{t}\right\|_{2}^{2}+\tilde{J}(u)=\frac{1}{2}\left\|u_{t}\right\|_{2}^{2}+\frac{1}{2}\|\nabla u\|_{2}^{2}-\int_{\Omega} G(u) d x \\
& \geq \frac{1}{2}\left\|u_{t}\right\|_{2}^{2}+\frac{1}{2}\|\nabla u\|_{2}^{2}-\frac{1}{\theta} \int_{\Omega} g(u) u d x \\
& \geq \frac{1}{2}\left\|u_{t}\right\|_{2}^{2}+\left(\frac{1}{2}-\frac{1}{\theta}\right)\|\nabla u\|_{2}^{2},
\end{aligned}
$$

for all $t \geq 0$. If $u=0$, (3.5) is obvious.

Lemma 3.2 (Energy decay) Under the assumptions imposed on Lemma 3.1, and suppose that there exists a constant $\eta_{0} \in(0,1)$ such that

$$
\tilde{I}(u(t)) \geq \eta_{0}\|\nabla u(t)\|_{2}^{2}, \quad \text { for } t \geq 0 .
$$

Then we have the energy $E(t)$ satisfies the decay estimates

$$
E(t) \leq I_{0} e^{-\kappa[t-1]^{+}}
$$

on $[0, \infty)$, where $I_{0}=E(0)$, and $\kappa$ is a positive constant.

Proof It follows from (1.5) and (1.8) that

$$
I(u(t))=\tilde{I}(u(t))+\|\nabla u(t)\|_{2}^{\gamma+2} .
$$

Hence, from Lemma 3.1 and (3.6), we deduce that

$$
I(u(t)) \geq \eta_{0}\|\nabla u(t)\|_{2}^{2}, \quad I(u(t)) \geq\|\nabla u(t)\|_{2}^{\gamma+2} \quad \text { on }[0, \infty) .
$$

Multiplying (1.1) by $u_{t}$ and integrating over $[t, t+1] \times \Omega$, we obtain

$$
\int_{t}^{t+1}\left\|u_{t}(s)\right\|_{2}^{2} d s=E(t)-E(t+1) \equiv D(t)^{2} .
$$


Thus, there exist two numbers $t_{1} \in\left[t, t+\frac{1}{4}\right]$ and $t_{2} \in\left[t+\frac{3}{4}, t+1\right]$ such that

$$
\left\|u_{t}\left(t_{i}\right)\right\|_{2} \leq 2 D(t) \quad(i=1,2) .
$$

Multiplying (1.1) by $u$ and integrating over $\left[t_{1}, t_{2}\right] \times \Omega$, we obtain

$$
\int_{t_{1}}^{t_{2}} I(u(s)) d s=\int_{t_{1}}^{t_{2}}\left\|u_{t}\right\|_{2}^{2} d s+\left(u\left(t_{1}\right), u_{t}\left(t_{1}\right)\right)-\left(u\left(t_{2}\right), u_{t}\left(t_{2}\right)\right)+\int_{t_{1}}^{t_{2}}\left(u_{t}(s), u(s)\right) d s .
$$

Combining (3.5), (3.9), (3.10), and $E(t)$ being nonincreasing, we deduce

$$
\int_{t_{1}}^{t_{2}} I(u(s)) d s \leq D(t)^{2}+c_{1} D(t) E(t)^{\frac{1}{2}}
$$

where $c_{1}=5 \sqrt{\frac{2 \theta}{(\theta-2) \lambda_{1}}}$. On the other hand, from (1.7) and (3.8) we obtain

$$
J(u(t))=\frac{1}{2}\|\nabla u\|_{2}^{2}+\frac{1}{\gamma+2}\|\nabla u\|_{2}^{\gamma+2}-\int_{\Omega} G(u) d x \leq\left(\frac{1}{2 \eta_{0}}+\frac{1}{\gamma+2}\right) I(u) .
$$

Hence, combining (3.11) and (3.12), we get

$$
\int_{t_{1}}^{t_{2}} E(s) d s=\frac{1}{2} \int_{t_{1}}^{t_{2}}\left\|u_{t}(s)\right\|_{2}^{2} d s+\int_{t_{1}}^{t_{2}} J(u(s)) d s \leq c_{2}\left(D(t)^{2}+D(t) E(t)^{\frac{1}{2}}\right)
$$

where $c_{2}=c\left(\eta_{0}, \gamma, c_{1}\right)$. Since $t_{2}-t_{1} \geq \frac{1}{2}$, we get

$$
\int_{t_{1}}^{t_{2}} E(s) d s \geq \int_{t_{1}}^{t_{2}} E\left(t_{2}\right) d s \geq \frac{1}{2} E\left(t_{2}\right)
$$

Thus, from energy identity (3.2) and (3.13), we obtain

$$
\begin{aligned}
E(t) & =E\left(t_{2}\right)+\int_{t}^{t_{2}}\left\|u_{t}(s)\right\|_{2}^{2} d s \leq 2 \int_{t_{1}}^{t_{2}} E(s) d s+\int_{t}^{t+1}\left\|u_{t}(s)\right\|_{2}^{2} d s \\
& \leq c_{3}\left(D(t)^{2}+D(t) E(t)^{\frac{1}{2}}\right) \quad \text { on }[0, \infty),
\end{aligned}
$$

for some constant $c_{3}>1$. Hence, there exists a constant $c_{4}>1$ such that

$$
E(t) \leq c_{4} D(t)^{2}=c_{4}(E(t)-E(t+1)) \quad \text { on }[0, \infty)
$$

The application of Nakao's inequality [15] to (3.14) yields (3.7) with $\kappa=\log \left(c_{4} /\left(c_{4}-1\right)\right)$.

We are now in a position to obtain $H^{2}$ a priori bounds. Set

$$
\begin{aligned}
& E_{*}(t)=\left\|\nabla u_{t}(t)\right\|_{2}^{2}+M\left(\|\nabla u(t)\|_{2}^{2}\right)\|\Delta u(t)\|_{2}^{2}, \\
& I_{0}=E(0), \quad I_{1}=\left\|\nabla u_{1}\right\|_{2}^{2}+\left\|\Delta u_{0}\right\|_{2}^{2} .
\end{aligned}
$$


Lemma 3.3 ( $H^{2}$ a priori estimate) Under the assumptions imposed on Lemma 3.2. Suppose $u(t)$ is a local solution on $[0, T)$ such that $\sup \left\{\left\|\nabla u_{t}(t)\right\|_{2},\|\Delta u(t)\|_{2}\right\}<K$ on $[0, T)$ for some $K$ and $T>0$. Then we have the following estimate:

$$
E_{*}(t) \leq I_{1}+C_{1}\left(I_{0}\right) K^{\frac{3}{2}}+C_{2}\left(I_{0}\right) K^{2}+C_{3}\left(I_{0}\right) K^{3} \equiv G\left(I_{0}, I_{1}, K\right) \quad \text { on }[0, T),
$$

where $C_{i}\left(I_{0}\right)$ is a constant depending increasing on $I_{0}$ and $\lim _{I_{0} \rightarrow 0} G\left(I_{0}, I_{1}, K\right)=I_{1}(i=1,2,3)$.

Proof Multiplying (1.1) by $-\Delta u_{t}(t)$ and integrating over $\Omega$, we obtain

$$
\begin{aligned}
& \frac{1}{2} \frac{d}{d t}\left[\left\|\nabla u_{t}(t)\right\|_{2}^{2}+M\left(\|\nabla u(t)\|_{2}^{2}\right)\|\Delta u(t)\|_{2}^{2}\right]+\left\|\nabla u_{t}(t)\right\|_{2}^{2} \\
& \quad=M^{\prime}\left(\|\nabla u(t)\|_{2}^{2}\right)\left(\nabla u_{t}(t), \nabla u(t)\right)\|\Delta u(t)\|_{2}^{2}+\left(\nabla g\left(u(t), \nabla u_{t}(t)\right)\right) .
\end{aligned}
$$

It follows from Hölder's inequality and assumption (2.1) that the second term in the righthand side of (3.15) can be estimated as

$$
\begin{aligned}
\left|\left(\nabla g\left(u(t), \nabla u_{t}(t)\right)\right)\right| & \leq \int_{\Omega}\left|g^{\prime}(u(t)) \nabla u(t) \cdot \nabla u_{t}(t)\right| d x \\
& \leq C_{\beta}\left(\int_{\Omega} e^{6 \beta u^{2}} d x\right)^{\frac{1}{6}}\|\nabla u(t)\|_{3}\left\|\nabla u_{t}(t)\right\|_{2} .
\end{aligned}
$$

On the other hand from $\|\nabla u(t)\|_{2}^{2} \leq \frac{2 \theta d}{\theta-2} \doteq R^{2}$, we deduce from the Moser-Trudinger inequality that

$$
\begin{aligned}
\sup _{\|\nabla u\|_{2} \leq R} \int_{\Omega} e^{6 \beta u^{2}} d x & =\sup _{\|\nabla u\|_{2} \leq R} \int_{\Omega} e^{6 \beta\|\nabla u\|_{2}^{2}\left(\frac{u}{\|\nabla u\|_{2}}\right)^{2}} d x \\
& \leq \sup _{\|\nabla u\|_{2} \leq R} \int_{\Omega} e^{6 \beta R^{2}\left(\frac{u}{\|\nabla u\|_{2}}\right)^{2}} d x \leq c_{5}
\end{aligned}
$$

where $c_{5}$ is a positive constant, as long as we choose $\beta<\frac{2 \pi}{3 R^{2}}$.

Hence, Sobolev's inequality and the interpolation inequality imply

$$
\begin{aligned}
\left|\left(\nabla g\left(u(t), \nabla u_{t}(t)\right)\right)\right| & \leq C_{\beta} c_{5}^{\frac{1}{6}}\|\nabla u(t)\|_{3}\left\|\nabla u_{t}(t)\right\|_{2} \\
& \leq C_{\beta} c_{5}^{\frac{1}{6}}\|\nabla u\|_{2}^{\frac{1}{2}}\|\nabla u\|_{6}^{\frac{1}{2}}\left\|\nabla u_{t}(t)\right\|_{2} \\
& \leq c_{6} K^{\frac{3}{2}} E(t)^{\frac{1}{4}},
\end{aligned}
$$

for some positive constant $c_{6}$, where we have also used (3.5). The first term on the righthand side of (3.15) is estimated as

$$
\frac{\gamma}{2}\|\nabla u(t)\|_{2}^{\gamma-1}\left\|\nabla u_{t}(t)\right\|_{2}\|\Delta u(t)\|_{2}^{2} \leq c_{7} K^{3} E(t)^{\frac{\gamma-1}{2}},
$$

for some constant $c_{7}>0$. Thus, from (3.15), (3.16), and (3.17), we obtain

$$
\begin{aligned}
& \frac{1}{2} \frac{d}{d t}\left[\left\|\nabla u_{t}(t)\right\|_{2}^{2}+M\left(\|\nabla u(t)\|_{2}^{2}\right)\|\Delta u(t)\|_{2}^{2}\right]+\left\|\nabla u_{t}(t)\right\|_{2}^{2} \\
& \quad \leq c_{6} K^{\frac{3}{2}} E(t)^{\frac{1}{4}}+c_{7} K^{3} E(t)^{\frac{\gamma-1}{2}} .
\end{aligned}
$$


Integrating (3.18) over $[0, t]$, noticing $E_{*}(0) \leq I_{1}+c_{8} I_{0}^{\frac{\gamma}{2}} K^{2}$ for some $c_{8}>0$, we obtain

$$
\begin{aligned}
E_{*}(t) \leq & I_{1}+c_{8} K^{2} I_{0}^{\frac{\gamma}{2}}+2 c_{6} K^{\frac{3}{2}} \int_{0}^{t} E(s)^{\frac{1}{4}} d s+2 c_{7} K^{3} \int_{0}^{t} E(s)^{\frac{\gamma-1}{2}} d s \\
\leq & I_{1}+c_{8} K^{2} I_{0}^{\frac{\gamma}{2}}+2 c_{6} K^{\frac{3}{2}} I_{0}^{\frac{1}{4}}+2 c_{7} K^{3} I_{0}^{\frac{\gamma-1}{2}} \\
& +2 c_{6} K^{\frac{3}{2}} I_{0}^{\frac{1}{4}} \int_{0}^{\infty} \exp \left(-\frac{\kappa}{4} s\right) d s+2 c_{7} K^{3} I_{0}^{\frac{\gamma-1}{2}} \int_{0}^{\infty} \exp \left(-\frac{\kappa(\gamma-1)}{2} s\right) d s \\
\leq & I_{1}+c_{8} K^{2} I_{0}^{\frac{\gamma}{2}}+2 c_{6} K^{\frac{3}{2}} I_{0}^{\frac{1}{4}}+\frac{8 c_{6} K^{\frac{3}{2}} I_{0}^{\frac{1}{4}}}{\kappa}+\frac{4 c_{7} K^{3} I_{0}^{\frac{\gamma-1}{2}}}{\kappa(\gamma-1)} \\
\equiv & G\left(I_{0}, I_{1}, K\right) .
\end{aligned}
$$

Thus, we complete the proof of Lemma 3.3.

Let $K>0$ and put

$$
\begin{aligned}
& H\left(I_{0}, I_{1}, K\right)=G\left(I_{0}, I_{1}, K\right)^{\frac{1}{2}}, \\
& S_{K}=\left\{\left(u_{0}, u_{1}\right) \in W \cap H^{2}(\Omega) \times H_{0}^{1}(\Omega) \mid H\left(I_{0}, I_{1}, K\right)<K\right\}
\end{aligned}
$$

and

$$
S=\bigcup_{K>0} S_{K}
$$

By the same method as considered in [6], we can deduce that $S$ is an open unbounded set, and if $\left(u_{0}, u_{1}\right) \in S$, the solution $u(t)$ can be continued globally on $[0, \infty)$ and $\left(u(t), u_{t}(t)\right) \in S$ for all $t \geq 0$.

Uniqueness: Let $u(t)$ and $v(t)$ be two solutions; $w(t)=u(t)-v(t)$ satisfies

$$
\begin{aligned}
& w_{t t}-M\left(\|\nabla u(t)\|_{2}^{2}\right) \Delta w+w_{t}(t) \\
& \quad=\left(M\left(\|\nabla u(t)\|_{2}^{2}\right)-M\left(\|\nabla v(t)\|_{2}^{2}\right)\right) \Delta v+(g(u)-g(v)),
\end{aligned}
$$

with $w=0$ on $[0, \infty) \times \partial \Omega$ and $w(0)=w_{t}(0)=0$ in $\Omega$. Taking the $L^{2}(\Omega)$ inner product on both sides of (3.20) with $w_{t}$, we can easily find that

$$
\begin{aligned}
\frac{1}{2} & \frac{d}{d t}\left\{\left\|w_{t}\right\|_{2}^{2}+M\left(\|\nabla u(t)\|_{2}^{2}\right)\|\nabla w(t)\|_{2}^{2}\right\}+\left\|w_{t}\right\|_{2}^{2} \\
= & M^{\prime}\left(\|\nabla u(t)\|_{2}^{2}\right)\left(\nabla u, \nabla u_{t}\right)\|\nabla w\|_{2}^{2}+\left[M\left(\|\nabla u(t)\|_{2}^{2}\right)-M\left(\|\nabla v(t)\|_{2}^{2}\right)\right]\left(\Delta v, w_{t}\right) \\
& +\left(g(u)-g(v), w_{t}\right) .
\end{aligned}
$$

Using assumption (A1), or more precisely (2.1), we estimate the last term as

$$
\int_{\Omega}(g(u)-g(v)) w_{t} d x \leq C \int_{\Omega}\left(e^{\beta|u|^{2}+}+e^{\beta|v|^{2}}\right)\left|w(t) w_{t}(t)\right| d x .
$$

Since $u(t), v(t)$ are two solutions, from (3.5), we obtain $\|\nabla u(t)\|_{2}^{2} \leq \frac{2 \theta}{\theta-2} d,\|\nabla v(t)\|_{2}^{2} \leq \frac{2 \theta}{\theta-2} d$. Repeating a similar procedure as estimating the term $\left(\nabla g(u), \nabla u_{t}\right)$, after employing the 
Hölder and the Moser-Trudinger inequality, yields

$$
\int_{\Omega}(g(u)-g(v)) w_{t} d x \leq c\|\nabla w\|_{2}\left\|w_{t}\right\|_{2}
$$

On the hand the first and the second term on the right-hand side of (3.21) are bounded by

$$
c\|\nabla w(t)\|_{2}^{2}, \quad c\|\nabla w(t)\|_{2}\left\|w_{t}(t)\right\|_{2}
$$

respectively. Thus, integrating $(3.21)$ over $(0, t)$, we obtain

$$
\left\|w_{t}(t)\right\|_{2}^{2}+\left\|\nabla w_{t}(t)\right\|_{2}^{2} \leq c \int_{0}^{t}\left\{\left\|w_{t}(s)\right\|_{2}^{2}+\left\|\nabla w_{t}(s)\right\|_{2}^{2}\right\} d s
$$

which implies $w=0$ by Gronwall's inequality. Thus, we complete the proof of Theorem 2.1.

Remark 3.1 As is well known, the difficult for Kirchhoff equations is proving the approximate solutions converge to the desired solution. Indeed, we prove the local existence solution for the problem (1.1) by Picard's iteration method. To utilize the standard compactness argument for the limiting procedure, it suffices to derive some $a$ priori estimates for $u_{m}(t)$ (see Lemma 3.1 and Lemma 3.3). In this direction, we also mention [6] and [10].

\subsection{Proof of Theorem 2.2}

In this section, we will give the proof of Theorem 2.2, which is similar to the proof Theorem 2.1. We sketch it as follows.

Proof of Theorem 2.2 For brevity, we take the same notations $E(t), E_{*}(t), I(t)$, and $D(t)$ as in the proof of Theorem 2.1, but since $g(s) s \leq 0$ for all $t \in \mathbb{R}$, we can deduce

$$
\left\|u_{t}(t)\right\|_{2}^{2}+\|\nabla u(t)\|_{2}^{2}+2 \int_{0}^{t}\left\|u_{t}(s)\right\|_{2}^{2} d s \leq 2 E(t) \leq 2 E(0) \quad \text { on }[0, \infty)
$$

and

$$
\|\nabla u(t)\|_{2}^{2} \leq I(t), \quad \text { for all } t \geq 0
$$

Similar to the proof of Lemma 3.2, we have

$$
\begin{aligned}
& \int_{t_{1}}^{t_{2}} E(s) d s \\
& \quad=\frac{1}{2} \int_{t_{1}}^{t_{2}}\left\|u_{t}(s)\right\|_{2}^{2} d s+\frac{1}{2} \int_{t_{1}}^{t_{2}} \bar{M}\left(\|\nabla u(s)\|_{2}^{2}\right) d s-\int_{t_{1}}^{t_{2}} \int_{\Omega} G(u(s)) d x d s \\
& \quad \leq \frac{1}{2} \int_{t_{1}}^{t_{2}}\left\|u_{t}(s)\right\|_{2}^{2} d s+\frac{M_{0}}{2} \int_{t_{1}}^{t_{2}}\|\nabla u(s)\|_{2}^{2} d s-\int_{t_{1}}^{t_{2}} \int_{\Omega} G(u(s)) d x d s
\end{aligned}
$$

where $M_{0}=\max \{M(s): s \in[0,2 E(0)]\}$, which is possible since $M(s)$ is continuous and (3.22). Now, we only need to estimate the term $-\int_{t_{1}}^{t_{2}} \int_{\Omega} G(u(s)) d x d s$. Indeed, from (A1), 
or more precisely $(2.5)$, we have

$$
\begin{aligned}
& -\int_{t_{1}}^{t_{2}} \int_{\Omega} G(u(s)) d x d s \\
& \quad \leq \frac{\varepsilon}{2} \int_{t_{1}}^{t_{2}} \int_{\Omega}|u(s)|^{2} d x d s+C_{\beta, \varepsilon} \int_{t_{1}}^{t_{2}} \int_{\Omega}|u(s)|^{2} e^{\beta u^{2}} d x d s \\
& \quad \leq \frac{\varepsilon}{2 \lambda_{1}} \int_{t_{1}}^{t_{2}}\|\nabla u(s)\|_{2}^{2} d s+C_{\beta, \varepsilon} \int_{t_{1}}^{t_{2}}\|u(s)\|_{2}\|u(s)\|_{4}\left(\int_{\Omega} e^{4 \beta u(s)^{2}} d x\right)^{1 / 4} d s .
\end{aligned}
$$

From (3.22) and the Moser-Trudinger inequality, we have

$$
\begin{aligned}
& \sup _{\|\nabla u\|_{2}^{2} \leq 2 E(0)} \int_{\Omega} e^{4 \beta u^{2}} d x \\
& =\sup _{\|\nabla u\|_{2}^{2} \leq 2 E(0)} \int_{\Omega} e^{4 \beta\|\nabla u\|_{2}^{2}\left(\frac{u}{\|\nabla u\|_{2}}\right)^{2}} d x \\
& \leq \sup _{\|\nabla u\|_{2}^{2} \leq 2 E(0)} \int_{\Omega} e^{8 \beta E(0)\left(\frac{u}{\|\nabla u\|_{2}}\right)^{2}} d x \leq c,
\end{aligned}
$$

where $c$ is a positive constant, as long as we choose $\beta<\frac{\pi}{2 E(0)}$. Hence, by the Sobolev inequality, there exists a constant $c$ such that

$$
-\int_{t_{1}}^{t_{2}} \int_{\Omega} G(u(s)) d x d s \leq c \int_{t_{1}}^{t_{2}}\|\nabla u(s)\|_{2}^{2} d s .
$$

Combining (3.22)-(3.24) and (3.11), we have

$$
\int_{t_{1}}^{t_{2}} E(s) d s \leq c\left(D(t)^{2}+D(t) E(t)^{\frac{1}{2}}\right) .
$$

Then, by the same argument of Lemma 3.2 we can obtain the decay estimate

$$
E(t) \leq E(0) e^{-\kappa[t-1]^{+}} \quad \text { on }[0, \infty)
$$

where $\kappa$ is a positive constant. Hence, it suffices to show $H^{2}(\Omega)$ a priori bounds under the assumption $\|\nabla u(t)\|_{2}^{2} \leq K$, and $\|\Delta u(t)\|_{2} \leq K$ on $[0, T)$ for some $K>0$ and $T>0$. Set

$$
I_{2}=\left\|\nabla u_{1}\right\|_{2}^{2}+M\left(\left\|\nabla u_{0}\right\|_{2}^{2}\right)\left\|\Delta u_{0}\right\|_{2}^{2}
$$

By the same derivation as Lemma 3.2, using (A3), we deduce

$$
\begin{aligned}
E_{*}(t) \leq & I_{2}+2 \int_{0}^{t} M^{\prime}\left(\|\nabla u(s)\|_{2}^{2}\right)\left(\nabla u_{t}(s), \nabla u(s)\right)\|\Delta u(s)\|_{2}^{2} d s \\
& +2 \int_{0}^{t}\left|\left(\nabla g(u(s)), \nabla u_{t}(s)\right)\right| d s \\
\leq & I_{2}+2 K^{3} \int_{0}^{t} E(s)^{\frac{2 \alpha+1}{2}} d s+2 c_{6} K^{\frac{3}{2}} \int_{0}^{t} E(s)^{\frac{1}{4}} d s
\end{aligned}
$$




$$
\begin{aligned}
& \leq I_{2}+2 c_{6} K^{\frac{3}{2}} I_{0}^{\frac{1}{4}}+\frac{8 c_{6} K^{\frac{3}{2}} I_{0}^{\frac{1}{4}}}{\kappa}+\frac{4 K^{3} I_{0}^{\frac{2 \alpha+1}{2}}}{\kappa(2 \alpha+1)} \\
& \equiv G\left(I_{0}, I_{2}, K\right)
\end{aligned}
$$

Thus, we can prove Theorem 2.2 in the same way as Theorem 2.1.

Remark 3.2 When $M(s)=1,(1.1)$ is a wave equation, Alves and Cavalcanti [2] obtain the general energy decay result. Indeed, Lemma 3.3 (to be precise: (3.44)) in [2] plays an important role in the proof of energy decay, where the authors used the unique continuation property of wave equations; see [16] for details and $[2,17,18]$ for an application. But in our case, since $M\left(\|\nabla \cdot\|_{2}^{2}\right) \triangle$. is nonlinear, we cannot use the unique continuation property directly.

\section{The blow-up in finite time}

In this section, we shall discuss the blow-up properties for the problem (1.1). For this purpose, we use the following lemmas.

Lemma 4.1 ([19]) Let $\delta>0$ and $B(t) \in C^{2}(0, \infty)$ be a nonnegative function satisfying

$$
B^{\prime \prime}(t)-4(\delta+1) B^{\prime}(t)+4(\delta+1) B(t) \geq 0 .
$$

If

$$
B^{\prime}(t)>r_{2} B(0)+K_{0},
$$

then $B^{\prime}(t)>K_{0}$ for $t>0$, where $K_{0}$ is a constant, $r_{2}=2(\delta+1)-2 \sqrt{(\delta+1) \delta}$ is the smaller root of the equation

$$
r^{2}-4(\delta+1) r+4(\delta+1)=0 .
$$

Lemma 4.2 ([19]) If $J(t)$ is a nonincreasing function on $\left[t_{0}, \infty\right), t_{0} \geq 0$, and satisfies the differential inequality

$$
J^{\prime}(t)^{2} \geq a+b J(t)^{2+\frac{1}{\delta}}, \quad \text { for } t \geq 0
$$

where $a>0, b \in \mathbb{R}$, then there exists a finite time $T^{*}$ such that

$$
\lim _{t \rightarrow T^{*-}} J(t)=0
$$

and the upper bound of $T^{*}$ is estimated, respectively, by the following cases:

(i) If $b<0$ and $J\left(t_{0}\right)<\min \left\{1, \sqrt{\frac{a}{-b}}\right\}$, then $T^{*} \leq t_{0}+\frac{1}{\sqrt{-b}} \ln \frac{\sqrt{\frac{a}{-b}}}{\sqrt{\frac{a}{-b}}-J\left(t_{0}\right)}$.

(ii) If $b=0$, then $T^{*} \leq t_{0}+\frac{J\left(t_{0}\right)}{\sqrt{a}}$.

(iii) If $b>0$, then $T^{*} \leq \frac{J\left(t_{0}\right)}{\sqrt{a}}$ or $T^{*} \leq t_{0}+2^{\frac{3 \delta+1}{2 \delta}} \frac{\delta c}{\sqrt{a}}\left\{1-\left[1+c J\left(t_{0}\right)\right]^{\frac{-1}{2 \delta}}\right\}$, where $c=\left(\frac{b}{a}\right)^{\frac{\delta}{2+\delta}}$.

We also denote $I(u)$ and $E(t)$ by (1.8) and (1.9), respectively. Also $E^{\prime}(t)=-\left\|u_{t}(t)\right\|_{2}^{2}$. 
Definition 4.1 A solution $u(t)$ of (1.1) is called a blow-up solution if there exists a finite time $T^{*}$ such that

$$
\lim _{t \rightarrow T^{*-}} \int_{\Omega}|u|^{2} d x=+\infty
$$

For the next lemma, we define

$$
K(t):=K(u(t))=\|u(t)\|_{2}^{2}+\int_{0}^{t}\|u(s)\|_{2}^{2} d s, \quad t \geq 0 .
$$

Lemma 4.3 Assume that (A1) and (A4) hold, then we have

$$
K^{\prime \prime}(t)-4(\delta+1)\left\|u_{t}\right\|_{2}^{2} \geq(-4-8 \delta) E(0)+(4+8 \delta) \int_{0}^{t}\left\|u_{t}(s)\right\|_{2}^{2} d s .
$$

Proof From (4.4), we obtain

$$
K^{\prime}(t)=2 \int_{\Omega} u u_{t} d x+\|u(t)\|_{2}^{2}
$$

and

$$
K^{\prime \prime}(t)=2\left\|u_{t}\right\|_{2}^{2}-2 M\left(\|\nabla u\|_{2}^{2}\right)\|\nabla u\|_{2}^{2}+2 \int_{\Omega} g(u) u d x .
$$

From the above equation and the energy identity, we obtain

$$
\begin{aligned}
K^{\prime \prime}-4(\delta+1)\left\|u_{t}\right\|_{2}^{2}= & (-4-8 \delta) E(0)+(4+8 \delta) \int_{0}^{t}\left\|u_{t}(s)\right\|_{2}^{2} d s \\
& +\int_{\Omega} 2[f(u) u-(2+4 \delta) F(u)] d x \\
& +\left\{(2+4 \delta) \bar{M}\left(\|\nabla u(t)\|_{2}^{2}\right)-2 M\left(\|\nabla u(t)\|_{2}^{2}\right)\|\nabla u(t)\|_{2}^{2}\right\} .
\end{aligned}
$$

Therefore from the assumption (A4), we obtain (4.5).

Now, we consider three different cases on the sign of initial energy $E(0)$.

(1) If $E(0)<0$, then from (4.5), we have

$$
K^{\prime}(t) \geq K^{\prime}(0)-4(1+2 \delta) E(0) t, \quad t \geq 0 .
$$

Thus, we get $K^{\prime}(t)>\left\|u_{0}\right\|_{2}^{2}$ for $t>t^{*}$, where

$$
t^{*}=\max \left\{\frac{K^{\prime}(0)-\left\|u_{0}\right\|_{2}^{2}}{4(1+2 \delta) E(0)}, 0\right\} .
$$

(2) If $E(0)=0$, then $K^{\prime \prime}(t) \geq 0$ for $t \geq 0$. Furthermore, if $K^{\prime}(0)>\left\|u_{0}\right\|_{2}^{2}$, i.e. $\int_{\Omega} u_{0} u_{1} d x>0$. Then we get $K^{\prime}(t)>\left\|u_{0}\right\|_{2}^{2}$ for $t \geq 0$.

(3) For the case that $E(0)>0$, we first note that

$$
2 \int_{0}^{t} \int_{\Omega} u u_{t} d x d t=\|u(t)\|_{2}^{2}-\left\|u_{0}\right\|_{2}^{2}
$$


By the Hölder inequality and the Young inequality, we have from (4.8)

$$
\|u(t)\|_{2}^{2} \leq\left\|u_{0}\right\|_{2}^{2}+\int_{0}^{t}\|u(s)\|_{2}^{2} d s+\int_{0}^{t}\left\|u_{t}(s)\right\|_{2}^{2} d s
$$

Hence, from (4.8) and (4.9), we obtain

$$
K^{\prime}(t) \leq K(t)+\left\|u_{t}\right\|_{2}^{2}+\int_{0}^{t}\left\|u_{t}(s)\right\|_{2}^{2} d s+\left\|u_{0}\right\|_{2}^{2} .
$$

Then, from the above inequality and (4.5), we obtain

$$
K^{\prime \prime}(t)-4(1+\delta) K^{\prime}(t)+4(1+\delta) K(t)+K_{1} \geq 0,
$$

where

$$
K_{1}=(4+8 \delta) E(0)+4(1+\delta)\left\|u_{0}\right\|_{2}^{2}
$$

Set

$$
B(t)=K(t)+\frac{K_{1}}{4(1+\delta)}, \quad t>0
$$

Then $B(t)$ satisfies (4.1). From Lemma 4.1, we see that if

$$
K^{\prime}(0)>r_{2}\left[K(0)+\frac{K_{1}}{4(1+\delta)}\right]+\left\|u_{0}\right\|_{2}^{2}
$$

then $K^{\prime}(t)>\left\|u_{0}\right\|_{2}^{2}$ for all $t>0$.

Consequently, we obtain the following lemma.

Lemma 4.4 Assume that (A1) and (A4) hold and that either one of the following conditions is satisfied:

(i) $E(0)<0$,

(ii) $E(0)=0$ and $\int_{\Omega} u_{0} u_{1} d x>0$,

(iii) $E(0)>0$ and (4.10) holds, then $K^{\prime}(t)>\left\|u_{0}\right\|_{2}^{2}$ for $t>t_{0}$, where $t_{0}=t^{*}$ is given by (4.8) in case (i) and $t_{0}=0$ in cases (ii) and (iii).

Next, we will estimate the lifespan of $K(t)$ and prove Theorem 2.3.

Let

$$
J(t)=\left(K(t)+\left(T_{1}-t\right)\left\|u_{0}\right\|_{2}^{2}\right)^{-\delta}, \quad \text { for } t \in\left[0, T_{1}\right],
$$

where $T_{1}$ is some certain constant which will be chosen later. Then we get

$$
J^{\prime}(t)=-\delta J(t)^{1+\frac{1}{\delta}}\left(K^{\prime}(t)-\left\|u_{0}\right\|_{2}^{2}\right)
$$

and

$$
J^{\prime \prime}(t)=-\delta J(t)^{1+\frac{2}{\delta}} V(t)
$$


where

$$
V(t)=K^{\prime \prime}(t)\left[K(t)+\left(T_{1}-t\right)\left\|u_{0}\right\|_{2}^{2}\right]-(1+\delta)\left(K^{\prime}(t)-\left\|u_{0}\right\|_{2}^{2}\right) .
$$

For simplicity, we denote

$$
P=\|u(t)\|_{2}^{2}, \quad Q=\int_{0}^{t}\|u(s)\|_{2}^{2} d s, \quad R=\left\|u_{t}(t)\right\|_{2}^{2}, \quad S=\int_{0}^{t}\left\|u_{t}(s)\right\|_{2}^{2} d s
$$

By (4.9) and the Hölder inequality, we obtain

$$
\begin{aligned}
K^{\prime}(t) & =2 \int_{\Omega} u(t) u_{t}(t) d x+\left\|u_{0}\right\|_{2}^{2}+2 \int_{0}^{t} \int_{\Omega} u(s) u_{t}(s) d x d s \\
& \leq\left\|u_{0}\right\|_{2}^{2}+2(\sqrt{P R}+\sqrt{Q S})
\end{aligned}
$$

From (4.5), we have

$$
K^{\prime \prime}(t) \geq(-4-8 \delta) E(0)+4(1+\delta)(R+S) .
$$

Therefore, from (4.13)-(4.15), and then (4.11), we have

$$
\begin{aligned}
V(t) \geq & {[(-4-8 \delta) E(0)+4(1+\delta)(R+S)]\left(K(t)+\left(T_{1}-t\right)\left\|u_{0}\right\|_{2}^{2}\right) } \\
& -4(1+\delta)(\sqrt{P R}+\sqrt{Q S})^{2} \\
= & (-4-8 \delta) E(0) J(t)^{-\frac{1}{\delta}}+4(1+\delta)(R+S)\left(T_{1}-t\right)\left\|u_{0}\right\|_{2}^{2} \\
& +4(1+\delta)\left[(R+S)(P+Q)-(\sqrt{P R}+\sqrt{Q S})^{2}\right] \\
\geq & (-4-8 \delta) E(0) J(t)^{-\frac{1}{\delta}}, \quad t \geq t_{0},
\end{aligned}
$$

where we have used Schwarz inequality in the last but one term. Therefore from (4.12), we have

$$
J^{\prime \prime}(t) \leq \delta(4+8 \delta) E(0) J(t)^{1+\frac{1}{\delta}}, \quad t \geq t_{0}
$$

Note that by Lemma 4.1, $J^{\prime}(t)<0$ for $t>t_{0}$. Multiplying (4.16) by $J^{\prime}(t)$ and integrating it from $t_{0}$ to $t$, we have

$$
J^{\prime}(t)^{2} \geq \alpha+\beta J(t)^{2+\frac{1}{\delta},} \quad \text { for } t \geq t_{0}
$$

where

$$
\alpha=\delta^{2} J\left(t_{0}\right)^{2+\frac{2}{\delta}}\left[\left(K^{\prime}\left(t_{0}\right)-\left\|u_{0}\right\|_{2}^{2}\right)^{2}-8 E(0) J\left(t_{0}\right)^{-\frac{1}{\delta}}\right]
$$

and

$$
\beta=8 \delta^{2} E(0) .
$$


We observe that

$$
\alpha>0 \quad \text { if and only if } \quad E(0)<\frac{\left(K^{\prime}\left(t_{0}\right)-\left\|u_{0}\right\|_{2}^{2}\right)^{2}}{8\left[K\left(t_{0}\right)+\left(T_{1}-t_{0}\right)\left\|u_{0}\right\|_{2}^{2}\right]} .
$$

Then by Lemma 4.2, there exists a finite time $T^{*}$ such that $\lim _{t \rightarrow T^{*-}} J(t)=0$ and the upper bounds of $T^{*}$ are estimated, respectively, according to the sign of $E(0)$. This yields

$$
\lim _{t \rightarrow T^{*-}}\left(\|u(t)\|_{2}^{2}+\int_{0}^{t} \int_{\Omega} u(s)^{2} d x d s\right)=\infty
$$

The upper bounds of $T^{*}$ are estimated as follows by Lemma 4.2.

In case (i),

$$
T^{*} \leq t_{0}-\frac{J\left(t_{0}\right)}{J^{\prime}\left(t_{0}\right)}
$$

Furthermore, if $J\left(t_{0}\right)<\min \left\{1, \sqrt{\frac{\alpha}{-\beta}}\right\}$, then we have

$$
T^{*} \leq t_{0}+\frac{1}{\sqrt{-\beta}} \ln \frac{\sqrt{\frac{\alpha}{-\beta}}}{\sqrt{\frac{\alpha}{-\beta}}-J\left(t_{0}\right)} .
$$

In case (ii),

$$
T^{*} \leq t_{0}-\frac{J\left(t_{0}\right)}{J^{\prime}\left(t_{0}\right)} \quad \text { or } \quad T^{*} \leq t_{0}-\frac{J\left(t_{0}\right)}{\sqrt{\alpha}} .
$$

In case (iii),

$$
T^{*} \leq \frac{J\left(t_{0}\right)}{\sqrt{a}}
$$

or

$$
T^{*} \leq t_{0}+2^{\frac{3 \delta+1}{2 \delta}} \frac{\delta c}{\sqrt{\alpha}}\left\{1-\left[1+c J\left(t_{0}\right)\right]^{\frac{-1}{2 \delta}}\right\}
$$

where $c=\left(\frac{\beta}{\alpha}\right) \frac{\delta}{2+\delta}$, here $\alpha$ and $\beta$ are defined in (4.17) and (4.18), respectively. Note that in case (i), $t_{0}=t^{*}$ is given in (4.7), and in case (ii) and case (iii) $t_{0}=0$.

Remark 4.1 We observe that the choice of $T_{1}$ in (4.11) is feasible under the same conditions as in [9]. 


\section{Acknowledgements}

The author would like to thank the Prof. Hua Chen in Wuhan University for pertinent discussions. The author would also like to thank the referee for the careful reading of this paper and for the valuable suggestions to improve its presentation and style. This work is supported by the Doctor Foundation of Henan University of Technology (2012BS058) and Plan of Nature Science Fundamental Research in Henan University of Technology (No. 2013JCYJ11).

Received: 28 June 2014 Accepted: 3 October 2014 Published online: 23 October 2014

\section{References}

1. Kirchhoff, G: Vorlesungen über Mechanik. Teubner, Leipzig (1883)

2. Alves, CO, Cavalcanti, MM: On existence, uniform decay rates and blow up for solutions of the 2-D wave equation with exponential source. Calc. Var. Partial Differ. Equ. 34(3), 377-411 (2009)

3. Ma, TF, Soriano, JA: On weak solutions to an evolution equation with exponential nonlinearities. Nonlinear Anal., Theory Methods Appl. 37, 1029-1038 (1999)

4. Han, X, Wang, M: Well-posedness for the 2-D damped wave equations with exponential source terms. Math. Methods Appl. Sci. 17, 2087-2100 (2010)

5. Gazzola, F, Squassina, M: Global solutions and finite time blow up for semilinear wave equation. Ann. Inst. Henri Poincaré, Anal. Non Linéaire 23(2), 185-207 (2006)

6. Matsuyama, T, Ikehata, R: On global solution and energy decay for the wave equation of Kirchhoff type with nonlinear damping term. J. Math. Anal. Appl. 204, 729-753 (1996)

7. Ono, K: Global existence, decay and blowup of solutions for some mildly degenerate nonlinear Kirchhoff strings. J. Differ. Equ. 137, 273-301 (1997)

8. Ono, K: On global solutions and blow-up solutions of nonlinear Kirchhoff strings with nonlinear dissipation. J. Math. Anal. Appl. 216, 321-342 (1997)

9. Wu, S, Tsai, L: Blow-up of solutions for some nonlinear wave equations of Kirchhoff type with some dissipation. Nonlinear Anal., Theory Methods Appl. 65, 243-264 (2006)

10. $\mathrm{Wu}, \mathrm{S}, \mathrm{Tsai}, \mathrm{L}$ : On the existence and nonexistence of solutions for some nonlinear wave equations of Kirchhoff type. Taiwan. J. Math. 14(4), 1543-1570 (2010)

11. Ambrosetti, A, Rabinowitz, PH: Dual variational methods in critical point theory and applications. J. Funct. Anal. 14, 349-381 (1973)

12. Willem, M: Minimax Theorems. Progress in Nonlinear Differential Equations and Their Applications, vol. 24. Birkhäuser, Basel (1996)

13. Moser, J: A sharp form of an inequality by Trudinger. Indiana Univ. Math. J. 20, 1077-1092 (1971)

14. Trudinger, NS: On the imbeddings into Orlicz spaces and applications. J. Math. Mech. 17, 473-484 (1967)

15. Nakao, M: A difference inequality and its application to nonlinear evolution equations. J. Math. Soc. Jpn. 30(4), 747-762 (1978)

16. Ruiz, A: Unique continuation for weak solutions of the wave equation plus a potential. J. Math. Pures Appl. 71 , 455-467 (1992)

17. Cavalcanti, MM, Domingos Cavalcanti, VN, Martinez, P: Existence and decay rate estimates for the wave equation with nonlinear boundary damping and source term. J. Differ. Equ. 203(1), 119-158 (2004)

18. Lasiecka, I, Tataru, D: Uniform boundary stabilization of semilinear wave equation with nonlinear boundary damping. Differ. Integral Equ. 8, 507-533 (1993)

19. Li, M, Tsai, L: Existence and nonexistence of global solutions of some systems of semilinear wave equations. Nonlinear Anal., Theory Methods Appl. 54, 1397-1415 (2003)

doi:10.1186/s13661-014-0230-3

Cite this article as: Liu: On global solution, energy decay and blow-up for 2-D Kirchhoff equation with exponentia

terms. Boundary Value Problems 2014 2014:230

\section{Submit your manuscript to a SpringerOpen ${ }^{\circ}$ journal and benefit from:}

- Convenient online submission

Rigorous peer review

- Immediate publication on acceptance

- Open access: articles freely available online

- High visibility within the field

- Retaining the copyright to your article 